\title{
Magnetic Reconnection, Cosmic Ray Acceleration, and Gamma-Ray emission around Black Holes and Relativistic Jets
}

\author{
Elisabete M. de Gouveia Dal Pino* \\ Instituto de Astronomia, Geofísica e Ciências Atmosféricas - Universidade de São Paulo \\ (IAG/USP), São Paulo, Brazil \\ E-mail: dalpino@iag.usp.br
}

Rafael Alves Batista

IAG - Universidade de São Paulo, Brazil

\section{Luis S. Kadowaki}

IAG - Universidade de São Paulo, Brazil

\section{Grzergorz Kowal}

EACH - Universidade de São Paulo, and UniSul, Brazil

\section{Tania Medina-Torrejon}

Instituto de Fisica - Universidade de São Paulo, Brazil

\section{Juan Carlos Ramirez-Rodriguez}

IAG - Universidade de São Paulo, Brazil

\begin{abstract}
Particle acceleration by magnetic reconnection is now recognized as an important process in magnetically dominated regions of galactic and extragalactic black hole sources. This process helps to solve current puzzles specially related to the origin of the very high energy flare emission in these sources. In this review, we discuss this acceleration mechanism and show recent analytical studies and multidimensional numerical SRMHD and GRMHD (special and general relativistic magnetohydrodynamical) simulations with the injection of test particles, which help us to understand this process both in relativistic jets and coronal regions of these sources. The very high energy and neutrino emission resulting from the accelerated particles by reconnection is also discussed.
\end{abstract}

International Conference on Black Holes as Cosmic Batteries: UHECRs and Multimessenger Astronomy BHCB2018

12-15 September, 2018

Foz du Iguazu, Brasil

${ }^{*}$ Speaker. 


\section{Introduction}

The detection of gamma-ray flares at TeV energies in AGN blazars, like PKS2155-304 [3], and more recently, the simultaneous observation of an IceCube neutrino event and a gamma-ray flare in blazar TXS 0506+056 [40] are among the most puzzling discoveries in high-energy astrophysics.

Blazars, which are AGNs with highly beamed relativistic jets pointing to the line of sight are, in fact, the most common sources of $\gamma$-rays. Their strongly Doppler boosted non-thermal emission, with apparently very high fluxes, is power-law and usually attributed to relativistic particles accelerated stochastically in shocks along the jet. However, the observed very short duration of the flares (for instance, of a few minutes only, in the case of PKS2155-304), imply explosive and extremely compact acceleration/emission regions $\left(<R_{S} / c\right)$ with Lorentz factors much larger than the typical bulk values $(\Gamma \sim 5-10)$, in order to avoid electron-positron pair creation and therefore, a complete gamma-ray re-absorption within the source (e.g. [8]). The only mechanism able to explain this high variability and compactness of the $\mathrm{TeV}$ emission seems to be fast magnetic reconnection involving misaligned current sheets inside the jet [33,51]. A similar mechanism has been also invoked to explain the transition from magnetically to kinetically dominated flow and the prompt gamma-ray emission in gamma-ray-bursts (GRBs; [82]). Furthermore, the simultaneous detection of VHE gamma-ray flare and neutrino emission in TXS 0506+056 is suggestive of hadronic interactions between highly relativistic protons and background photons that result in pions and a subsequent decay in gamma-rays and neutrinos. If produced in the magnetically dominated regions of the blazar jet near the core, these protons are probably accelerated by magnetic reconnection [17].

Also puzzling is the recent detection by ground based gamma-ray observatories (e.g. [69] and references therein) of $\mathrm{TeV}$ fluxes from non-blazar sources (i.e. with misaligned jets with the line of sight), belonging to the low luminosity AGN branch for having bolometric luminosities of only a few times the Eddington luminosity (e.g. [69] and references therein). These sources (usually callled LLAGNs) include well studied radio galaxies like M87, Centaurus A, and Persus A. Upper limit detections in the $\mathrm{TeV}$ range from stellar mass galactic $\mathrm{BHs}$ of X-ray binary systems (also called BXBs), like Cyg X1 and X3, have been also reported ([4, 46] and references therein). The angular resolution and sensitivity of the current gamma-ray detectors are still so poor that it is hard to establish if this emission comes from the jet or from the coronal region around the black hole (BH). Fortunately, the forthcoming ground based Cherenkov Telescope Array (CTA) (see [1, 2]) will probably change this situation, but while it does not come, we may try at least to constrain the potential mechanisms.

In this regard, an interesting evidence has been found by Kadowaki, de Gouveia Dal Pino \& Singh (2015) [41] who plotted in the same diagram the $\gamma$-ray luminosity versus the BH mass for about 230 sources including non-blazar LLAGNs, black hole X-ray binaries (BXBs), blazars and GRBs, spanning $\sim 10$ orders of magnitude in mass and luminosity (see Figure 1). This diagram evidences two distinct branches, or correlation trends. One branch is followed by blazars and GRBs, and the other by LLAGNs and BXBs. The association between blazars and GRBs is already expected as their non-thermal radiation is commonly attributed to Doppler boosted emission by accelerated particles along the relativistic jets (e.g. [60]) (as discussed above). But the lack of correlation of these sources with non-blazars and BXBs in this diagram (see Figure 1) suggests that 
another location and/or population of relativistic particles may be producing the $\gamma$-ray emission in the latters. In earlier work, de Gouveia Dal Pino \& Lazarian (2005) [18] and de Gouveia Dal Pino et al. (2010) [19, 20], inspired by the physical processes in the solar corona, had proposed a non-thermal emission model based on the acceleration of particles by magnetic reconnection in the coronal region of accreting BH sources. This model, revisited in Kadowaki et al. (2015 [41]; see also [66]), have demonstrated that the magnetic reconnection power released in fast events, driven by local turbulence embedded in the large scale coronal magnetic lines, matches quite well with the observed $\gamma$-ray luminosity of the non-blazar LLAGNs and BXBs, plotted in the luminosity-BH mass diagram described above (see right panel of Figure 1, and [41, 66]). This result has been found to be nearly independent of the detailed physics of the accretion flow and suggests that magnetic reconnection occurring in the core region of these sources can in principle explain the observed $\gamma$-ray emission. Though other studies have attempted to explain these observations as produced in the jets of these sources (e.g. [70,53]), the evidences above led to a more detailed investigation of particle acceleration scenarios involving the production of the VHE in the coronal region of the accreting BH (see [41, 46, 44] and references therein). If such environments are magnetically dominated, as generally believed, then fast magnetic reconnection could be unavoidable, natural, and even efficient [18, 16, 21, 75].

In this lecture, we discuss briefly particle acceleration by fast magnetic reconnection and its potential role on the production of VHE in the surrounds of BH sources and along their relativistic jets. We will also argue about neutrino emission counterparts. Recent reviews on this subject can be also found in $[16,21,17,75]$ to mention a few.

\section{Particle Acceleration by Fast Magnetic Reconnection}

There is fast magnetic reconnection when two magnetic fluxes of opposite polarity encounter each other and partially annihilate at an efficient rate $V_{R}$ which is a substantial fraction of the local Alfvén speed $\left(V_{A}\right)$. Besides successful laboratory reconnection experiments [78] and direct observations in the earth magnetotail and in the solar corona flares, extensive numerical work has been also carried out to understand the nature of this process, both in collisionless (see, e.g. [72] for a review) and collisional plasmas (e.g., $[48,55]$ ). Different processes such as plasma instabilities, anomalous resistivity, and turbulence, can lead to fast reconnection. The latter process is very efficient and probably the main driving mechanism of fast reconnection in collisional MHD flows $[52,30,71]$. Turbulence causes the wandering of the magnetic field lines which allows for many independent patches to reconnect simultaneously making the reconnection rate large and independent on the local existing microscopic magnetic resistivity, $V_{R} \sim v_{A}\left(l_{\text {inj }} / L\right)^{1 / 2}\left(v_{\text {turb }} / v_{A}\right)^{2}$, where $l_{\text {inj }}$ and $v_{\text {turb }}$ are the injection scale and velocity of the turbulence, respectively [52].

The break of the magnetic field topoloy by fast reconnection involves the release of magnetic energy explosively which explains, for instance, the bursty emission in solar flares, but it can also explain flares in compact sources like pulsar magnetospheres and winds, relativistic jets and $\mathrm{BH}$ coronae. Relativistic particles are always observed in connection with these flares suggesting that magnetic reconnection can lead to direct particle acceleration [16].

de Gouveia Dal Pino \& Lazarian [18] seem to have been the first to realize that particles could be accelerated in magnetic reconnection sites in a similar way as in shocks where particles confined 

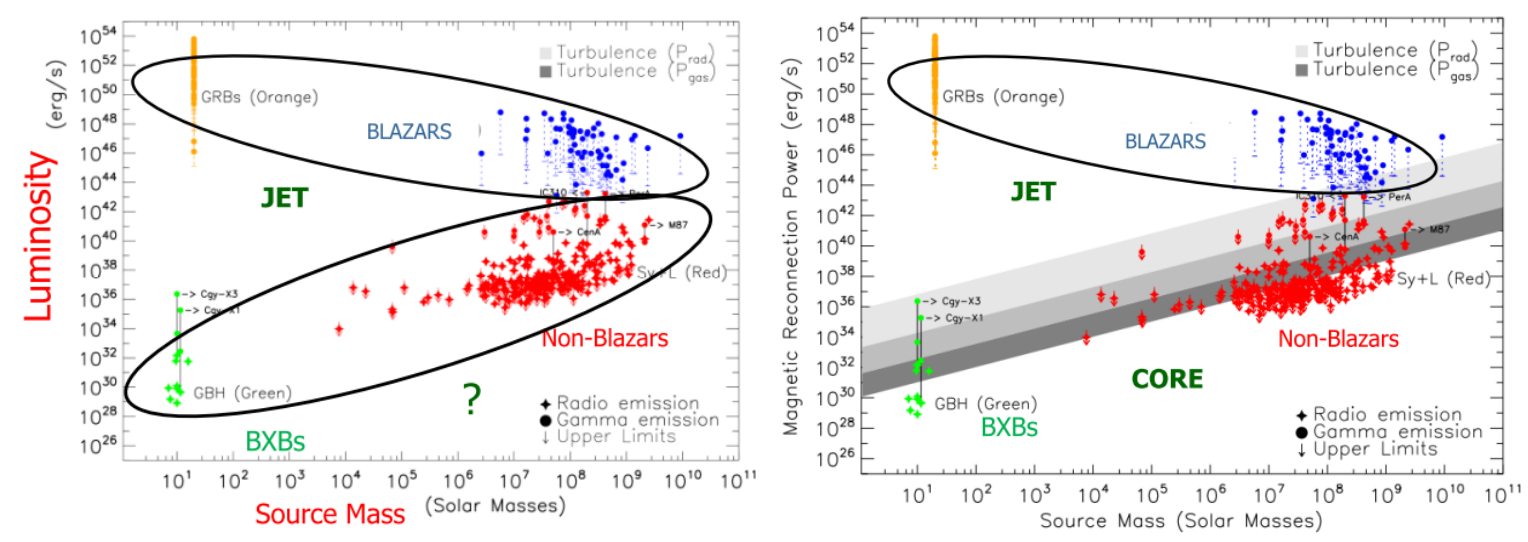

Figure 1: Observed radio and VHE emission of low luminous AGNs (LLAGNs, identified as non-blazars in the diagrams), galactic black hole binaries (BXBs, also labeled as GBHs in the diagrams), high luminous AGNs (blazars) and gamma ray burst (GRBs) versus BH source mass (symbols), compared to the calculated turbulent driven magnetic reconnection power (gray region in the right diagram). The core radio emission of the BXBs (or GBHs) and non-blazars (or LLAGNs) is represented by red and green diamonds, the gammaray emission of these two classes is represented by red and green circles, respectively. In the fewer sources for which there is observed gamma-ray luminosity it is plotted the maximum and minimum values linking both circles with a vertical black line that extends down to the radio emission of each of these sources. The inverted arrows associated to some sources indicate upper limits in gamma-ray emission. For blazars and GRBs, only the gamma-ray emission is depicted, represented in blue and orange circles, respectively. The vertical dashed lines correct the observed emission by Doppler boosting effects. The calculated magnetic reconnection power clearly matches the observed radio and gamma-ray emissions from LLAGNs (nonblazars) and GBHs (or GXBs), but not that from blazars and GRBs. This result confirms early evidences that the emission in blazars and GRBs is produced along the jet and not in the core of the sources, but on the other hand, it does indicate that the gamma-ray emission of LLAGNs (non-blazars) and GBHs (or BXBs) can be instead produced in the core region around the BH by magnetic reconnection processes (modified from $[41,66])$.

between the upstream and downstream flows undergo a first-order Fermi acceleration. Likewise, trapped particles are also able to bounce back and forth between the two converging magnetic fluxes of a reconnection discontinuity (or current sheet), gyrorotate around the reconnected magnetic field (see Figure 2 in [49], gaining energy due to collisions with magnetic fluctuations at a rate $\Delta E / E \propto V_{R} / c$ implying a first-order Fermi process with an exponential energy growth after several round trips [18]. In Kowal et al. (2011) [49], we have demonstrated the equivalence between this process and that of particles being accelerated while confined within merging magnetic islands, as often seen in two-dimensional reconnection flows.

The recognition of reconnection acceleration as a key mechanism to explain observed nonthermal, highly variable emission, specially at VHEs, in magnetically dominated sources, as described in Section 1, has made this a field of intensive theoretical and numerical study mainly through two-dimensional particle-in-cell (PIC) simulations of collisionless plasmas [27, 79, 80, 81, $57,28,14,13,54,56,75,76]$, and more recently also through three-dimensional (3D) PIC simulations $[68,36,37]$. However, these simulations, though essential in most aspects, can probe acceleration only at the kinetic scales of the plasma, of a few hundreds of the inertial length $\left(\sim 100 c / \omega_{p}\right.$, 
where $\omega_{p}$ is the plasma frequency). To assess the Fermi process in the large scales of the collisional MHD flows commonly observed in astrophyisical systems, the tracking of test particle distributions in such flows is a very useful and complementary tool to help in the understanding of the overall process through the scales. Such studies have been also successfully tested both in 2D and 3D MHD simulations with the injection of thousands of test particles in the reconnection domain by our and other groups [49, 50, 22, 61, 29, 34, 25].

Our recent studies, in particular, have revealed that test protons injected in large scale 3D MHD current sheets with turbulence embedded in order to make reconnection fast [50, 22], undergo efficient particle acceleration with a rate $t_{a c c}^{-1} \propto E^{-\alpha}$, with $0.2<\alpha<0.6$, for a vast range of values of $c / V_{\mathrm{A}} \sim 20-1000$. The accelerated particles produce power-law spectra with indices in the initial times of the simulations $N(E) \propto E^{-1,-2}$, where $E$ is the particle kinetic energy [22]. These powerlaw indices are compatible with the values derived in PIC studies of highly relativistic magnetized, non-radiative plasmas, both of electron-positron pairs (e.g, [35, 36, 74]) and electron-ion pairs (e.g., $[38,75,6])^{1}$. These studies have also demonstrated the important property that the reconnection acceleration rate is nearly independent of the intrinsic parameters that drive the turbulence and thus the fast reconnection in the collisional MHD flow. This is consistent with the notion that the turbulence is just the driving mechanism of fast reconnection and should not influence much the acceleration mechanism that is universal!

The results summarized above are general and in principle applicable to a vast amount of magnetized astrophysical flows. In the following paragraphs, we will discuss their applications to relativistic jets and coronal accreting plasma around $\mathrm{BH}$ sources, highlighting interesting properties found in our recent studies. I also refer to the contributions of Kadowaki et al. (2019) [43] and Ramirez-Rodriguez et al. (2019) [64] in these Proceedings.

\section{Magnetic Reconnection Acceleration and VHE in Relativistic Jets}

The origin of relativistic jets near central BHs is still uncertain, but the usually most accepted models rely on magnetic processes, like magneto-centrifugal acceleration by helical magnetic fields arising from the accretion disk [10]. Alternatively, they can be powered by the $\mathrm{BH}$ spin transferred to the surrounding magnetic flow ([11]; see also Blandford 2019 in these Proceedings [9]). Thus in any case the prediction is that the jets should be born as magnetically or Poynting flux dominated flows (see also [17]). The fact that at observable distances from the source (starting around $1000 R_{S}$ or less, where $R_{S}$ is the Schwartzschield radius) these jets become kinetically dominated, indicate that they should suffer efficient conversion (or dissipation) of magnetic into kinetic energy. It has been argued by several authors, including ourselves (e.g., $[16,12])$ that magnetic reconnection may be a powerful mechanism operating in these jets to allow for this conversion.

To probe this process, we performed 3D relativistic MHD simulations of rotating Poyinting flux dominated tower jets with initial helical fields [67]. Considering models with a ratio between

\footnotetext{
${ }^{1}$ We remind that reconnection in electron-ion plasmas should be identical to that in electron-positron plasmas in a highly relativistic regime, since the energies become much larger than the rest mass energies both for electrons and ions in this case. In the mild or semi-relativistic case, where electrons are highly relativistic and ions weakly relativistic, the PIC simulations of Werner et al. (2018) [75], for instance, have revealed steeper power-law indices for the electron spectra, varying from 2 to 4 , as the plasma magnetization is decreased.
} 
the magnetic and the rest mass energy of the flow $\sigma=1$, and different density ratios between the jet and the environment, we induced precession perturbations that quickly developed currentdriven kink (CDK) modes [59, 15]. Figure 2 depicts a snapshot of a tower jet with density larger than that of the environment, after the propagation of a helical kinked structure driven by the CDK instability. This causes substantial distortion of the original straight plasma column leading to break and reconnection of the magnetic fields lines in several regions. The loci of maximum current density in this example are labeled with red circles. They trace the current-sheets where turbulent fast magnetic reconnection occurs with rates $\sim(0.01-0.15) V_{A}([67,43])$. We have employed the algorithm developed in Kadowaki et al. (2018) [42] to localize these current sheets (see more details in Kadowaki et al. 2019 in these Proceedings [43]). These values are compatible with those of recent studies of fast turbulent relativistic reconnection in MHD current sheets [71] and also in collisionless PIC simulations [75, 7]. Moreover, these values produce similar reconnection rates as in non-relativisitc flows [48, 42].

Figure 3 shows the time evolution of the volume averaged magnetic and kinetic energy densities of the jet flow of Figure 2. We see that the CDK instability and the magnetic reconnection lead to release and dissipation of magnetic energy accompanied by exponential increase of the kinetic energy density up to saturation (see also [67]). We have also found that this maximum growth of the kinetic energy coincides with the maximum growth of the rate of magnetic reconnection around the time interval $t=40$ in code units (see Figure 3 of Kadowaki et al. 2019 in these Proceedings [43]).

As discussed in Section 2, these fast reconnection regions are potential sites for Fermi particle acceleration. To probe this, we have performed in situ test particle acceleration injecting 1000 particles with initial Maxwellian velocity distribution into the relativistic jet of Figure 2 (see left diagram). Starting with energies $\sim 1 \mathrm{MeV}$, the particles undergo an exponential acceleration when they are trapped in the reconnection sheets and interact resonantly with plasma magnetic field fluctuations (as predicted in [18], and successfully tested in [49, 50, 22]), until a saturation level when then the accelerated particles Larmor radius become larger than the acceleration regions. The initial background magnetic field in this simulation had a maximum initial value $B=0.13 \mathrm{G}$, but we have also considered simulations with values about 100 times larger. In the latter case, when the particles enter the exponential regime, they are accelerated up to energies $\sim 10^{19} \mathrm{eV}$ at sub-pc scales for an AGN jet ([58]).

The implications of these results are specially important for blazar jets, as argued in Section 1. These accelerated protons have energy enough to produce VHE $\gamma$-rays within very compact regions as well as neutrinos, as recently detected in the blazar TXS 0506+056 [40], a scenario that deserves further detailed investigation in forthcoming work.

\section{Magnetic reconnection acceleration and VHE around black holes}

As stressed in Section 1, fast magnetic reconnection can also occur in magnetized accretion flows around the BH sources in both BXBs and AGNs and this has been investigated by several authors (e.g., [31, 24, 18, 73, 19, 20, 39, 46, 41, 66]).

We have obtained direct evidence of this process from general relativistic MHD (GRMHD) simulations of torus accretion disks around BHs, using the ATHENA ++ code [77], as shown in 


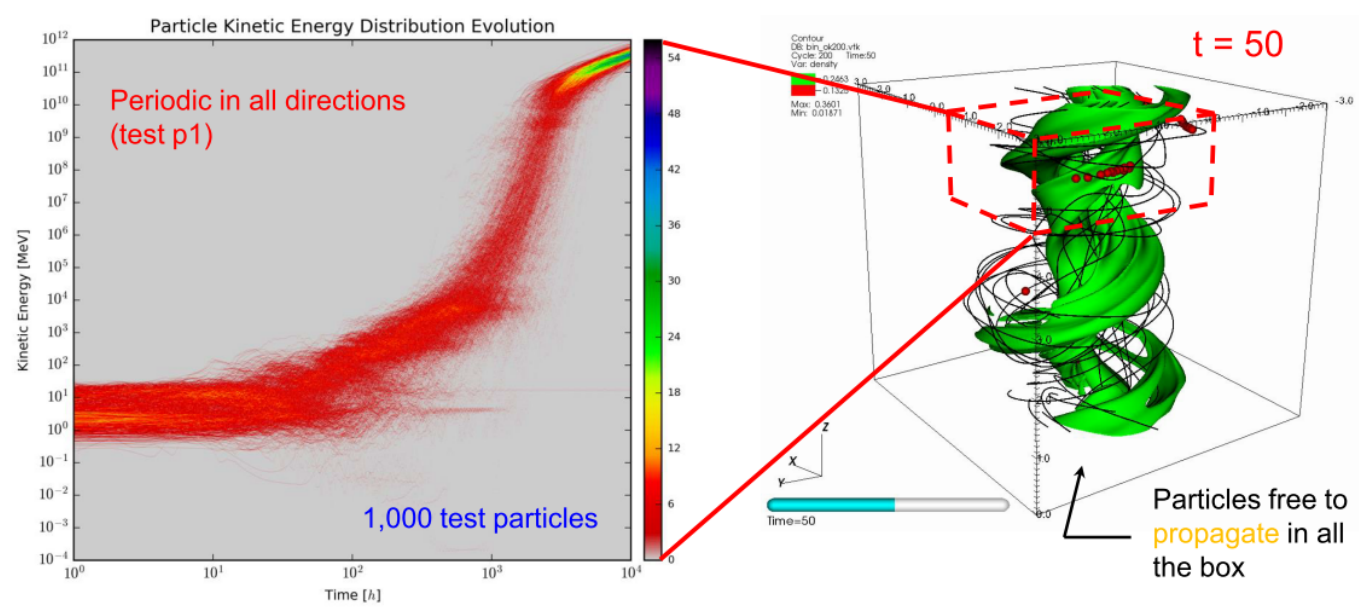

Figure 2: The right diagram depicts 3D density isosurfaces (green) with superposed magnetic field lines (black) of a tower jet at time $t=50$ in code units $(L / c)$ subject to the CDK instability that has distorted the originally straight plasma column with a helical magnetic field. The red circles identify the sites of fast reconnection driven by the CDK turbulence, employing the technique described in Kadowaki, de Gouveia Dal Pino \& Stone (2018 [42]; see also [43]). In this simulation, we considered a rotating jet with angular velocity equal 2 in code units, and initial maximum magnetic field intensity $B=0.13 G$. The boundaries are periodic in the z-direction, and outflow in the $\mathrm{x}$ and $\mathrm{y}$ directions. The grid resolution is $L / 40$, where $\mathrm{L}$ is the length scale code unit. The velocity code unit is the light speed, and the magnetic field is in units of $\left(4 \pi \rho_{o} c^{2}\right)^{1 / 2}$, where $\rho_{o}$ is the ambient density, taken as one code unit. The left diagram depicts the time evolution of the kinetic energy of 1000 test particles (protons) injected with an initial Maxwellian velocity distribution at the marked zone of the jet with a large concentration of current sheets (assuming periodic boundaries for the particles in all directions). Once particles are trapped within the reconnection regions they are exponentially accelerated up to energies $\sim 10^{17} \mathrm{eV}$ in this model. Simulations performed with magnetic fields one-hundred times larger, allow for particle acceleration up to $10^{19} \mathrm{eV}$ [58].

Figure 2 of Kadowaki et al. (2019 in these Proceedings [43]; see also [62, 23, 47, 17, 42, 7]). Moreover, employing a similar algorithm for current sheet search as described in Section 3 ([42, 43]), we could indentify several fast reconnection events in the turbulent accretion flow, with rates between 0.01 and 0.7 in Alfvén velocity units. This is the first time this method is applied in GRMHD simulations (see more details in Kadowaki et al. 2019 in these Proceedings [43]), and it demonstrates the efficiency of the reconnection process, as well its potential to accelerate particles and produce the VHE emission.

Considering the approximate magnetic reconnection power that is produced in the accreting flow around a BH, as calculated analytically in Kadowaki et al. [41] and Singh et al. [66], we computed the spectral energy distribution (SED) of a number of BXBs [46] and LLAGNs [44, 45] that lie in the "core" branch of Figure 1, aiming at explaining the origin of the VHE gammaray emission in these sources. Employing simple one-zone models to calculate the non-thermal leptonic and hadronic emission of the accelerated particles by reconnection, we could match the observed spectrum and in particular, the VHE flux at $\mathrm{TeV}$ energies of the investigated sources ([46, 44, 45]). Nevertheless, these simple models, though elucidating in many aspects, did not account for the spatial distribution of the background fields nor provided an appropriate calculation 

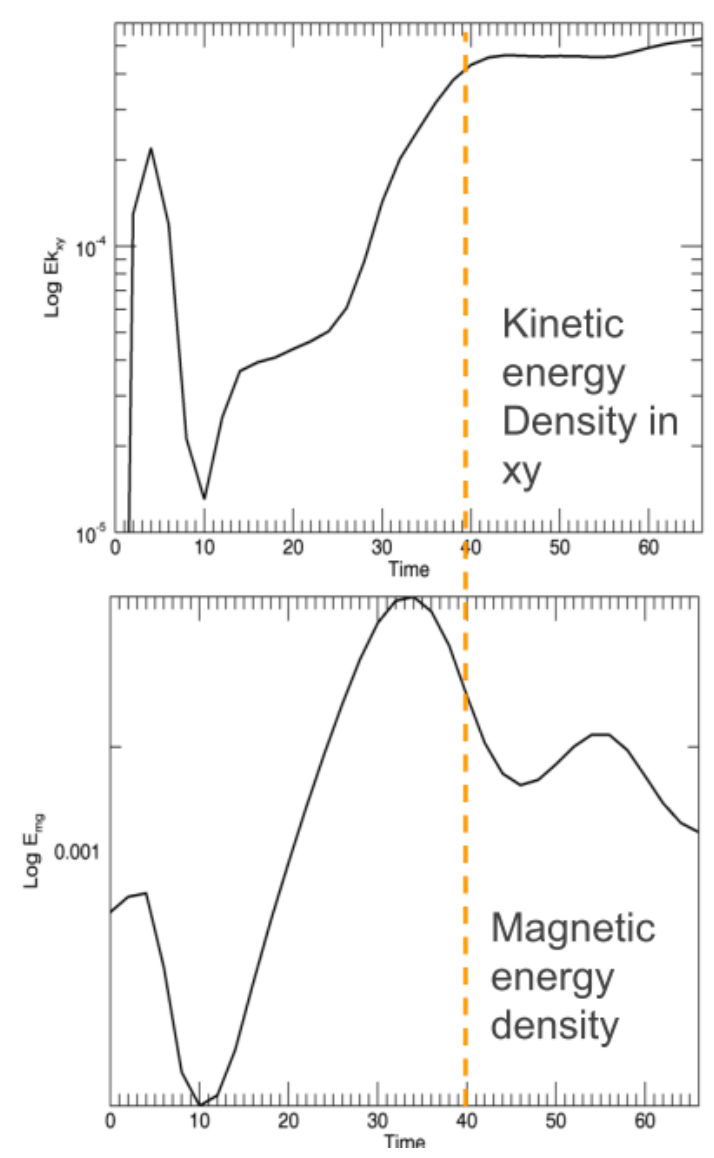

Figure 3: Volume-averaged time evolution of the kinetic (top) and magnetic (bottom) energy densities for the jet model presented in Figure 2 ([58]; see also [67]).

of the gamma-ray absorption by electron-positron pair production.

We are currently developing more detailed models, combining three different techniques (see Figure 4 and also Ramirez-Rodriguez et al. 2019 in these Proceedings [64]). First, we perform 2D GRMHD simulations of an accretion torus (using here the HARM code [32]) which allows us to obtain the density and magnetic field space distribution in the accretion flow around the $\mathrm{BH}$ (left column in Figure 4). Then, we employ post-processing radiative transfer calculation (employing the GRMONTY code [26]) to obtain the background photon field due to leptonic (electronic) Synchrotron and Invervse Compton emissions (middle column in Figure 4). Having the space distributions of these three background fields, we inject a power-law distribution of relativistic protons accelerated by magnetic reconnection according to the model described in Singh et al. (2015) [66]. The interactions of these protons with the background fields, calculated using the CRpropa code ([5]; see right column of Figure 4), allow us to obtain the space distributions and integrated fluxes of the hadronic emission, as well as the electron-positron pair creation and $\gamma$-ray absorption, and the neutrino emission. The application of this analysis to Per A AGN [63], Cen A [64], and to our galactic center Sgr A* [65], have demonstrated that the TeV emission in these sources can be explained by proton-proton and proton-photon interactions involving accelerated protons 
by reconnection. More important, we have found that this emission is not entirely absorbed by electron-positron pairs, as suspected before in earlier studies of these sources. Furthermore, the same process leads to neutrino emission too which can be compared directly with the observed extragalactic diffuse emission by the IceCube (see [45]). The details of this approach and the resulting SED for CenA as an example, are described in detail in Ramirez-Rodriguez et al. 2019, in these Proceedings [64]).

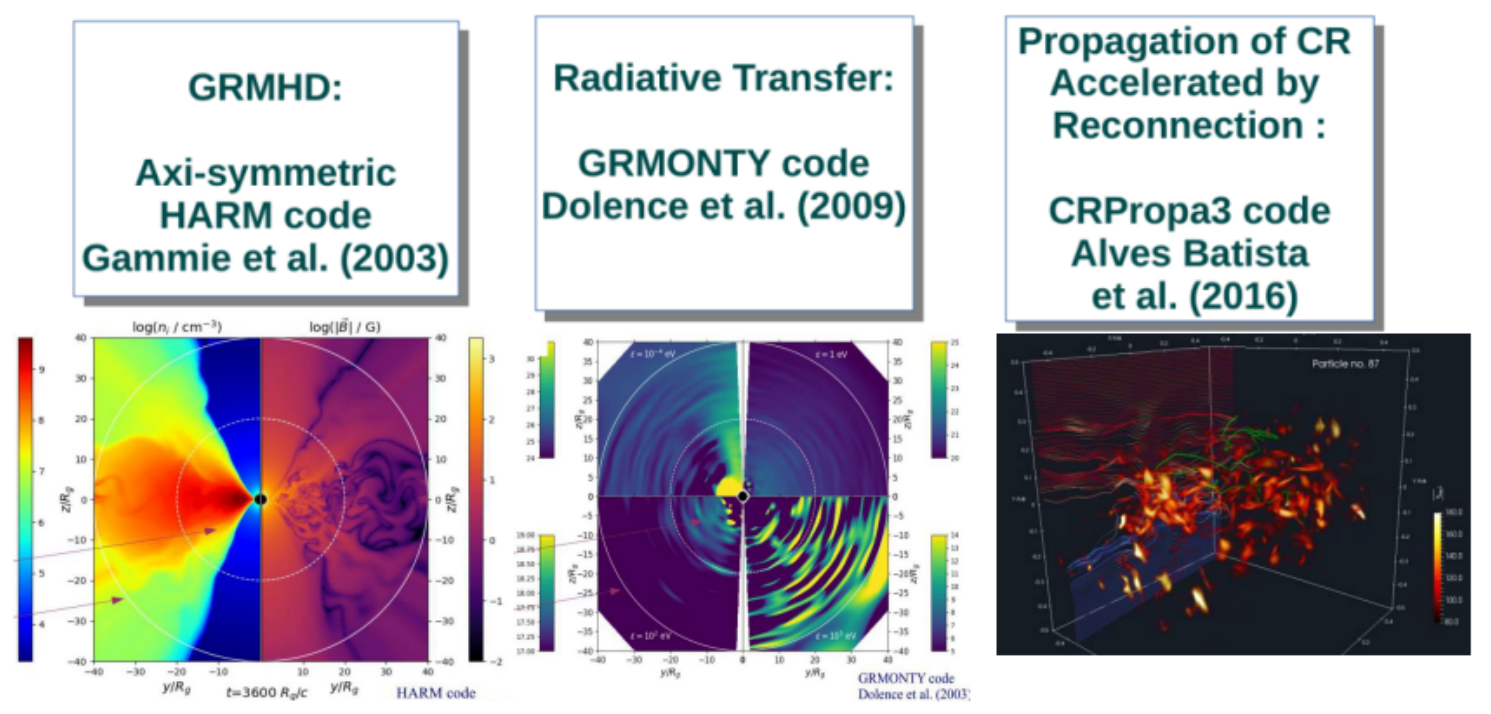

Figure 4: Schematic representation illustrating the combination of three different techniques to calculate the spectral energy distribution (SED) around BH sources. Left column depicts the density and magnetic field distribution of a 2D GRMHD simulation of a torus accreting flow around a BH where turbulence induced by magneto-rotational instability drives magnetic reconnection (as shown in Kadowaki et al. 2019, these Proceedings [43]). Middle column depicts the leptonic radiation field resulting from a radiative transfer simulation of photons in the background of the accreting flow of the left diagram. Each quadrant shows a photon energy distribution, in the clockwise sense from the top-left: $10^{-1} \mathrm{eV}, 1 \mathrm{eV}, 100 \mathrm{eV}$, and $10^{5} \mathrm{eV}$ [63]. Right column shows the trajectory of a single test particle (green line) being accelerated within a single current sheet by reconnected magnetic fields driven by turbulence [22]. A similar process should happen with an entire distribution of particles injected in the accretion flow depicted in the left column that contains several regions like this. We then injected a distribution of accelerated protons by reconnection in the inner circle (described in the left diagram by a white line) and computed the spatial hadronic radiative losses distribution over the domain (contained within the outer white circle depicted in the left diagram). See more details in $[64,65]$.

\section{Summary and Conclusions}

In this lecture, we have discussed the importance of turbulent magnetic reconnection and particle acceleration by reconnection in $\mathrm{BH}$ coronal regions and along relativistic jets based on studies supported by theory and global numerical simulations of collisional MHD flows. In general lines, our results are consistent and complementary to those found in collisionless local plasma studies of non-relativistic and relativistic reconnection, as stressed in Sections 1 and 2, but they have also 
revealed new aspects which are relevant at the large scales of these flows. Our main conclusions can be summarized as follows (see also [17]):

- Fast magnetic reconnection driven by instabilities and turbulence can be an important process in accretion and jet systems to allow for an efficient dissipation of magnetic energy, and conversion into kinetic energy and particle acceleration.

- In magnetized plasmas, particles can be predominantly accelerated by fast magnetic reconnection (driven e.g. by turbulence) subject to a Fermi-like process, and develop a power-law spectrum $N(E) \sim E^{-2,-1}[50,22]$. Steeper power-law indices can result, particularly if radiative processes are present.

- The magnetic reconnection power released in fast events occurring in the accretion flow around BHs $[42,43]$ could explain the observed $\gamma$-ray emission in non-blazar LLAGNs and BXBs. This power matches well with the observed correlation of $\gamma$-ray luminosity versus BH mass for these sources that spans 10 orders of magnitude [41, 66]. We have also found that accelerated CRs by magnetic reconnection in the core regions of these sources can produce $\mathrm{TeV} \gamma$-rays via hadronic processes which are not entirely re-absorbed by electronpositron pair production, as thought before. The same process also leads to neutrino production $[63,64,65]$.

- In relativistic jets (as in blazars, GRBs and BXBs), magnetic reconnection acceleration may be also very efficient $[67,43]$. For instance, our numerical simulations of test particles injected in relativistic MHD tower jets have revealed the production of cosmic rays with energies up to $10^{17} \mathrm{eV}$ and $10^{19} \mathrm{eV}$, for background magnetic fields around $\sim 0.13 \mathrm{G}$ and $\sim 13 \mathrm{G}$, respectively [58]. These protons have energy enough to be responsible for the $\gamma$-ray flares and neutrino emission in the magnetically dominated regions of these jets. This may be, for instance, the dominating mechanism responsible for the recent observed $\gamma$-ray and neutrino emission in the TXS 0506+056 blazar. This will be examined in detail in forthcoming work.

\section{Acknowledgments}

We acknowledge support from the Brazilian agencies FAPESP (2013/10559-5 grant) and CNPq (grant 308643/2017-8). The simulations presented in this lecture have made use of the computing facilities of the GAPAE group (IAG-USP) and the Laboratory of Astroinformatics IAG/USP, NAT/Unicsul (FAPESP grant 2009/54006-4).

\section{References}

[1] Actis, M. et al. (CTA collaboration) 2011, Exp. Astron., 32, 193

[2] Acharya, B.S. et al. (CTA collaboration) 2013, Astropart. Phys., 43, 3

[3] Aharonian, F. A., et al. 2007, The Astrophysical Journal, 664, L71

[4] Aleksić, J., Antonelli, L. A., Antoranz, P., et al. 2010, ApJ, 721, 843 
[5] Alves Batista, R., Dundovic, A., Erdmann, M., Kampert, K.-H., Kuempel, D., Müller, G., Sigl, G., van Vliet, A., Walz, D., \& Winchen, T. , 2016, Jcap, 5, 038

[6] Ball, D., Sironi, L. \& Özel, F. 2018a, ApJ, 862, 80

[7] Ball, D., Özel, F., Psaltis, D., Chan, C.-K. \& Sironi, L. 2018b, ApJ, 853, 184

[8] Begelman, M. C., Fabian, A. C. \& Rees, M. J. 2008, MNRAS, 384, L19

[9] Blandford, R. D., Meier, D. \& Readhead, A. 2018, arXiv:1812.06025

[10] Blandford, R. D., \& Payne, D. G. 1982, MNRAS, 199, 883

[11] Blandford, R. D., \& Znajek, R. L. 1977, MNRAS, 179, 433

[12] Bromberg, O. \& Tchekhovskoy, A. 2016, MNRAS, 456, 1739

[13] Cerutti, B., Uzdensky, D. A. \& Begelman, M. C. 2014, ApJ, 746, 148

[14] Clausen-Brown, E. \& Lyutikov, M. 2012, MNRAS, 426, 1374

[15] Das, U., \& Begelman, M. C. 2018, arXiv:1807.11480

[16] de Gouveia Dal Pino, E. M., \& Kowal, G. 2015, in Magnetic Fields in Diffuse Media, Astrophysics and Space Science Library, Eds. A. Lazarian. E. de Gouveia Dal Pino, C. Melioli, 407, 373

[17] de Gouveia Dal Pino, E. M., Kowal, G., Kadowaki, L., Medina Torrejón, T. E., Mizuno, Y. \& Singh, C., 2018, arXiv:1809.06742v1

[18] de Gouveia Dal Pino, E. M., \& Lazarian, A. 2005, A\&A, 441, 845 (GL05)

[19] de Gouveia Dal Pino, E. M., Piovezan, P. P. \& Kadowaki, L. H. S. 2010a, A\&A, 518, A5

[20] de Gouveia Dal Pino, E. M., Piovezan, P. P., Kadowaki, L. H. S., Kowal, G. \& Lazarian, A. 2010b, Highlights of Astronomy, 15, 247

[21] de Gouveia Dal Pino, E., del Valle, M. V., Kadowaki, L., Khiali, B., Kowal, G., Mizuno, Y. \& Singh, C. B. 2016, arXiv:1608.03173

[22] del Valle, M. V., de Gouveia Dal Pino, E.M., \& Kowal, G. 2016, MNRAS, 463, 4331

[23] Dexter, J., McKinney, J. C., Markoff, S., \& Tchekhovskoy, A. 2014, MNRAS, 440, 218

[24] Di Matteo, T. 1998, MNRAS, 299, L15

[25] Ding J., Yuan F., \& Liang E., 2010, ApJ, 708, 1545

[26] Dolence, J. C., Gammie, C. F., Mościbrodzka, M. \& Leung, P. K. 2009, ApJs, 184, 387

[27] Drake, J. F., Swisdak, M., Che, H., \& Shay, M. A. 2006, Nature, 443, 553

[28] Drake, J. F., Opher, M., Swisdak, M, \& Chamoun, J. N. 2010, ApJ, 709, 963

[29] Drake, J. F., Cassak, P. A., Shay, M. A., Swisdak, M. \& Quataert, E. 2009, ApJL, 700, L16

[30] Eyink, G. L., Lazarian, A. \& Vishniac, E. T. 2011, ApJ, 743, 51

[31] Galeev, A. A., Rosner, R. \& Vaiana, G. S. 1979, ApJ, 229, 318

[32] Gammie, C. F., McKinney, J. C. \& Tóth, G. 2003, ApJ, 589, 444

[33] Giannios, D., Uzdensky, D. A. \& Begelman, M. C., 2009, MNRAS, 228, 395 L29-L33

[34] Gordovskyy, M., Browning, P. K. \& Vekstein, G. E. 2010, ApJ, 720, 1603 
[35] Guo, F., Li, H., Daughton, W. \& Liu, Y. H., 2014, Physical Review Letters, 113, 155005

[36] Guo, F., Liu, Y. H., Daughton, W. \& Li, H. 2015, ApJ, 806, 167

[37] Guo, F., Li, H., Daughton, W., Li, X. \& Liu, Y.-H. 2016, Physics of Plasmas, 23, 055708

[38] Guo, F., Li, X., Li, H., et al. 2016, ApJL, 818, L9

[39] Hoshino, M. \& Lyubarsky, Y. 2012, Space Science Reviews, 173, 521

[40] IceCube Collaboration 2018, Science, 361, 147

[41] Kadowaki, L. H. S., de Gouveia Dal Pino, E. M., \& Singh, C. B. 2015, ApJ, 802, 113

[42] Kadowaki, L. H. S., de Gouveia Dal Pino, E. M. \& Stone, J. M. 2018, ApJ, 864, 52

[43] Kadowaki, L. H. S., de Gouveia Dal Pino, E. M., \& Medina-Torrejon, T. E. 2019, these Proceedings

[44] Khiali, B., de Gouveia Dal Pino, E. M. \& Sol, H. 2016, A\&A, arXiv:1504.07592

[45] Khiali, B. \& de Gouveia Dal Pino, E. M. 2016, MNRAS, 455, 838

[46] Khiali, B., de Gouveia Dal Pino, E. M., \& del Valle, M. V. 2015, MNRAS, 449, 34

[47] Koide, S. \& Arai, K. 2008, ApJ, 682, 1124

[48] Kowal, G., Lazarian, A., Vishniac, E. T., \& Otmianowska-Mazur, K., 2009, ApJ, 700, 63

[49] Kowal, G., de Gouveia Dal Pino, E. M., \& Lazarian, A. 2011, ApJ, 735, 102

[50] Kowal, G., de Gouveia Dal Pino, E. M., \& Lazarian, A. 2012, PRL, 108, 241102

[51] Kushwaha, P., Sinha, A., Misra, R., Singh, K. P., de Gouveia Dal Pino, E. M. et al. 2017, ApJ, 849, 138

[52] Lazarian, A. \& Vishniac, E. T. 1999, ApJ, 517, 700

[53] Lenain, J. P., Boisson, C., Sol, H. \& Katarzynski, K. 2008 A\&A, 478, 111

[54] Li, X., Guo, F., Li, H., \& Li, G. 2015, ApJ Lett., 811, L24

[55] Loureiro, N. F., Schekochihin, A. A., \& Cowley, S. C. 2007, Physics of Plasmas, 14, 100703

[56] Lyutikov, M., Sironi, L., Komissarov, S. S. \& Porth, O., 2017, Journal of Plasma Physics, 83, 635830602

[57] Lyubarsky, Y. \& Liverts, M. 2008, ApJ, 682, 1436

[58] Medina-Torrejon, T. E., de Gouveia Dal Pino, E. M., Kowal, G., Mizuno, Y., Singh, C., \& Kadowaki, L. 2019 (in prep.)

[59] Mizuno, Y., Lyubarsky, Y., Nishikawa, K.-I., \& Hardee, P. E. 2012, ApJ, 757, 16

[60] Nemmen, R. S., Georganopoulos, M., Guiriec, S., Meyer, E. T., Gehrels, N. \& Sambruna, R. M. 2012, Science, 338, 1445

[61] Onofri, M., Isliker, H. \& Vlahos, L. 2006, Physical Review Letters, 96, 151102

[62] Parfrey, K., Giannios, D., Beloborodov, A. M. 2015, MNRAS, 446, L61

[63] Rodriguez-Ramirez, J. C., de Gouveia Dal Pino, E. M., \& Alves Batista, R. 2018, arXiv:1811.02812

[64] Rodriguez-Ramirez, J. C., de Gouveia Dal Pino, E. M., \& Alves Batista, R. 2019, these Proceedings

[65] Rodriguez-Ramirez, J. C., de Gouveia Dal Pino, E. M., \& Alves Batista, R. 2019 (submitted) 
[66] Singh, C. B., de Gouveia Dal Pino, E. M., \& Kadowaki, L. H. S. 2015, ApJ Letts., 799, L20

[67] Singh, C. B., Mizuno, Y., \& de Gouveia Dal Pino, E. M. 2016, ApJ, 824, 48

[68] Sironi, L., \& Spitkovsky, A. 2014, ApJ Letts., 783, L21

[69] Sol, H., Zech, A., Boisson, C. et al. 2013, Astroparticle Physics, 43, 215

[70] Tavecchio F., Ghisellini G., 2008, MNRAS, 385, L98

[71] Takamoto, M., Inoue, T., \& Lazarian, A. 2015, ApJ, 815, 16

[72] Uzdensky, D. A. 2015, arXiv:1510.05397

[73] Uzdensky, D. A. \& Goodman, J. 2008, ApJ, 682, 608

[74] Werner, G. R., Uzdensky, D. A., Cerutti, B., Nalewajko, K. \& Begelman, M. C. 2016, ApJL, 816, L8

[75] Werner, G. R., Uzdensky, D. A., Begelman, M. C., Cerutti, B., \& Nalewajko, K. 2018, MNRAS, 473, 4840

[76] Werner, G. R., Philippov, A. A. \& Uzdensky, D. A. , 2019, MNRAS, 482, L60

[77] White, C. J., Stone, J. M. \& Gammie, C. F. 2016, ApJs, 225, 22

[78] Yamada, M., Kulsrud, R., \& Ji, H. 2010, Reviews of Modern Physics, 82, 603

[79] Zenitani, S. \& Hoshino, M. 2001, ApJ, 562L, 63Z

[80] Zenitani, S. \& Hoshino, M. 2007, ApJ, 670, 702

[81] Zenitani, S. \& Hoshino, M. 2008, AJ, 677, 530

[82] Zhang, B., \& Yan, H. 2011, ApJ, 726, 90 